\title{
New acute treatments for headache
}

\author{
Alan M. Rapoport
}

(C) The Author(s) 2010. This article is published with open access at Springerlink.com

\begin{abstract}
Although we have several acute care medications for the treatment of migraine, we are always looking for new medications to treat our patients. Patients often say that their headaches are not under optimal control and would be happy to try another medication. Patients look for faster onset of relief, more complete relief, no recurrent headache and no adverse events. This article will cover some new and some anticipated acute care products, CGRP antagonists, sumatriptan by iontophoretic patch, sumatriptan by needle-free injections, DHE by oral inhalation and diclofenac potassium in a sachet. Botulinum toxin therapy, although a preventive measure, will be mentioned at the end.
\end{abstract}

Keywords Acute treatment of migraine .

Migraine therapy - CGRP antagonist - Iontophoretic patch . Needle free injection · Sumatriptan · DHE · Oral inhalation

\section{CGRP receptor antagonists}

Calcitonin gene-related peptide, closely related structurally to calcitonin and amylin, has been intensely studied over the last 20 years as an agent possibly related to migraine pathophysiology. CGRP is involved in sensory neurotransmission and can be found in most sensory nerves, especially those trigeminovascular afferents in the meninges involved in migraine [1,2]. It is one of the most potent vasodilators known. CGRP levels measured in the jugular venous system are elevated after migraine and cluster

A. M. Rapoport ( $\square)$

The David Geffen School of Medicine at UCLA,

Los Angeles, CA, USA

e-mail: alanrapoport@gmail.com headache attacks, and are normalized by therapy with sumatriptan. For years, it was thought that blocking its dilating effect might help in treating migraine and its antagonism held promise to be a novel strategy to relieve migraine headache. It is now known to effectively block migraine pain without overt vasoconstriction. If and when they gain approval in the USA by the FDA and in other countries, CGRP receptor antagonists would be the first non-serotonergic, non-vasoconstricting, migraine-specific medication. CGRP has been shown to have several sites of action, including blood vessels, mast cells in the meninges and as a facilitator of pain transmission the brain stem [3]. CGRP receptors have been found in trigeminal ganglion, in the brain stem in neurons of the trigeminal nucleus caudalis and in smooth muscle of the meningeal vasculature [6]. CGRP can be blocked by a fragment of the peptide containing amino acids 8-37 (CGRP 8-37).

The first effective CGRP receptor blocker was BIBN4096 (olcegepant). It was reported that intravenous administration helped a significant number of patients versus placebo, without constricting blood vessels in preclinical studies [4]. Telcagepant, previously termed MK-0974, was the first reported oral formulation of a CGRP receptor antagonist. It has been reported to work well in migraine in a phase IIB study published in $\mathrm{Neu}$ rology and recently in a phase III study published in Lancet $[5,6]$. Preclinical data suggest that telcagepant is not a vasoconstrictor, and clinical studies show it to be as effective as rizatriptan and zolmitriptan and as tolerable as placebo. It is predicted that this drug could be launched in 2012. It was found to be as effective as zolmitriptan with fewer adverse events [6].

This was a randomized, parallel treatment, placebo and active-controlled, double-blind, trial performed at 81 sites in Europe and the USA of adults with migraine diagnosed 
by International Headache Society criteria. Patients treated moderate or severe migraine attacks with either oral telcagepant 150 or $300 \mathrm{mg}$, zolmitriptan $5 \mathrm{mg}$, or placebo. There were five co-primary endpoints: pain freedom, pain relief and absence of nausea, photophobia and phonophobia, at 2-h post treatment. According to Dr. Ho's article, “ 1,380 patients were randomly assigned to receive telcagepant $150 \mathrm{mg}(n=333), 300 \mathrm{mg}(n=354)$, zolmitriptan $5 \mathrm{mg}(n=345)$ or placebo $(n=348)$. Telcagepant $300 \mathrm{mg}$ was more effective than placebo for pain freedom [95 (27\%) of 353 patients vs. $33(10 \%)$ of 343 $(p=0.0001)]$, pain relief [194 (55\%) of 353 vs. $95(28 \%)$ of $343(p=0.0001)]$, and absence of phonophobia [204 $(58 \%)$ of 353 vs. $126(37 \%)$ of $342(p=0.0001)]$, photophobia $[180(51 \%)$ of 353 vs. $99 \quad(29 \%)$ of 342 $(p=0.0001)]$, and nausea [229 $(65 \%)$ of 352 vs. 189 $(55 \%)$ of $342(p=0.0061)]$. The efficacy of telcagepant $300 \mathrm{mg}$ and zolmitriptan $5 \mathrm{mg}$ was much the same, and both were more effective than telcagepant $150 \mathrm{mg}$. Adverse events were recorded for $31 \%$ taking telcagepant $150 \mathrm{mg}, 37 \%$ taking telcagepant $300 \mathrm{mg}, 51 \%$ taking zolmitriptan $5 \mathrm{mg}$, and $32 \%$ taking placebo [7]." The measurement of 2-24 h sustained pain freedom was slightly better numerically for telcagepant $300 \mathrm{mg}$ versus zolmitriptan. This drug may be able to be given to patients with vascular disease, but that was not studied in this trial as zolmitriptan is contraindicated in patients with vascular disease and this will have to be studied in the future.

\section{Transdermal patches}

Recently, sumatriptan became the first of the seven triptans to become generic in several countries, which has led to the development of generic formulations of available products and to the design of some novel products containing the generic formulation, including needle-free injection, sublingual, intranasal and patch forms. One of the most interesting products in development, which may address the unmet need of the nauseated migraineur and/or the patient who does not absorb oral medication optimally during a migraine attack, is a sumatriptan patch. NP101, which will be marketed as Zelrix from NuPathe, is an iontophoretic patch that delivers sumatriptan transdermally. It utilizes a small electric current to drive sumatriptan across the skin delivering 6 or $12 \mathrm{~mA} / \mathrm{h}$ and maintaining sumatriptan plasma levels above the target level of $10 \mathrm{ng} / \mathrm{ml}$ for greater than $7 \mathrm{~h} \mathrm{[8].} \mathrm{There} \mathrm{is} \mathrm{a} \mathrm{linear} \mathrm{relationship} \mathrm{between} \mathrm{the}$ applied current and drug delivery. As a result, drug delivery is precisely controlled at desired levels, providing consistent therapeutic drug levels. In $\mathrm{pK}$ studies, the patch delivered sumatriptan more consistently than either the $100 \mathrm{mg}$ oral tablet or $20 \mathrm{mg}$ nasal preparation. This finding supports the hypothesis that parenteral administration (subcutaneous or transdermal) provides more predictable delivery by bypassing absorption through the GI tract.

At the intended plasma concentrations delivered by the patch, which were in between those of the $20 \mathrm{mg}$ nasal spray and $100 \mathrm{mg}$ oral tablet, the patches were well tolerated. No subject reported atypical pain and pressure sensations or other common triptan adverse events after related pruritus, which was generally mild and resolved without treatment. No subject withdrew from the study due to local skin irritation. The data suggest that transdermal iontophoretic delivery of sumatriptan with NP101 may offer significant clinical utility for migraine patients, including circumventing underlying migraine-associated GI disturbances including nausea and gastric stasis. The patch also provides consistent, predictable delivery of desired drug levels over a 4-h period. This offers the potential to avoid atypical pain, pressure and other sensations commonly associated with current triptan formulations. The results of a recent phase-III trial were released at the American Headache Society meeting in Philadelphia. The 2-h pain relief was $53 \%$ versus $29 \%$ placebo. This was significant at $p=0.0001$ level. The drug also achieved significance over placebo in the three other parts of the primary endpoint, nausea free, photophobia free and sonophobia free. The pain free rate at $2 \mathrm{~h}$ was 18 versus $9 \%$ for placebo. The sustained pain relief rate from $2-24 \mathrm{~h}$ was $34 \%$ for the drug and $21 \%$ for placebo. Therefore, with good 2-h pain relief, relief of associated symptoms, few adverse events and the ability to bypass the GI tract, this should be an attractive alternative to standard oral treatment.

\section{Sumatriptan by needle-free injection}

Sumavel DosePro by Zogenix will deliver $6 \mathrm{mg}$ of sumatriptan subcutaneously through the skin without the use of a needle. The device is carefully designed to work exactly the same as the sumatriptan injection. Thus, it has excellent 1- and 2-h pain relief numbers. It is a sophisticated device that produces a small hole in the skin by the force of the injection using nitrogen gas fired through a tiny hole in the glass. The injection takes less than one tenth of a second. This system was marketed in the USA in January 2010, and is a good alternative for patients who are concerned about the needle and those who do not get adequate relieve from oral triptans.

\section{Oral inhaler for DHE}

DHE (dihydroergotamine mesylate) is being developed for delivery by oral inhalation in a novel, breath-actuated 
inhaler. It has been available in various forms for more than 50 years and still remains the mainstay of treatment at major headache centers in the USA via the intravenous route when patients require having already developed central sensitization. It is usually given three times per day intravenously. It is also used orally in Europe as a preventive and intranasally in the USA and Canada as an acute care medication. The intravenous preparation is often very effective, but cannot be used at home and many patients become nauseated or even vomit, despite pretreatment with an antiemetic. Oral inhalation seems to provide similar efficacy with the ease of home use and fewer adverse events.

Studies were performed with a specially designed device called the Tempo Inhaler (MAP Pharma), to deliver DHE deep into the lung after breath actuation [9]. The drug and device will be called Levadex. A phase I study of four doses of orally inhaled DHE delivered by the specially designed inhaler versus $1 \mathrm{mg}$ of IV DHE $(n=18)$ was performed. There was a rapid systemic absorption of DHE with a $t_{\max }$ of $12 \mathrm{~min}$ with a $0.88 \mathrm{mg}$ respirable dose (vs. a 6-min $t_{\max }$ with the IV preparation). The systemic levels attained were slightly lower than with IV DHE, with the ratio of AUC 0 to infinity of inhaled versus IV approximately 0.77 . The Tempo inhaler is a proprietary, novel, breath-actuated device that minimizes oropharyngeal deposition and therefore swallowing of the drug. Phase II data shows DHE gives relief that is both rapid and sustained [10]. Phase II results demonstrate that $32 \%$ of patients achieve pain relief as early as $10 \min (p=0.019)$ at $0.5 \mathrm{mg}$ dose. This is somewhat lower that the usual dose IV. DHE delivered by this inhaler was well tolerated in phase II studies with no serious adverse events. There was decreased nausea and no clinically significant changes observed in pulmonary function tests, clinical laboratory findings, heart rate, blood pressure or respiratory rate.

Phase III results presented in Philadelphia at the International Headache Congress in September 2009 showed a 2-h headache relief of 59 versus $35 \%$ placebo. This was significant at $p=0.0001$ level. The $2-24 \mathrm{~h}$ sustained headache relief rate was $44 \%$ for Levadex and $20 \%$ for placebo and the 2-48 h sustained pain free rate was 36 versus $17 \%$. Adverse events were low and nausea and vomiting surprisingly low (4.5 vs. $2 \%$ for nausea and 2 vs. $0.1 \%$ for vomiting). This low level in comparison to IV DHE probably is related to a lower $c_{\max }$. Other important findings were efficacy of Levadex given more than $8 \mathrm{~h}$ after the start of the headache, 49 versus $25 \%$ for the placebo and efficacy in disabling migraine, early morning migraine and menstrually related migraine.

Levadex is expected to get FDA approval in 2011 and should be a good drug for patients not responding well to oral triptans or for patients who are more than $6-8 \mathrm{~h}$ into their migraine attack.

\section{Diclofenac potassium for oral solution}

Cambia was approved for acute treatment of migraine in June 2009 and will be marketed in the spring of 2010 by Nautilus Neurosciences. It comes in a small packet or sachet and its contents are dissolved in water. As it is in solution, it gains rapid entry into the small intestine and has a faster $t_{\max }$ and onset of action than its tablet form. The 2-h pain relief is 65 versus $41 \%$ for placebo. Cambia will be the only NSAID approved for the acute treatment of migraine and could be an excellent drug for milder migraines, especially for patients with more frequent headaches.

\section{Neurotoxin therapy: botulinum toxin injections}

Although the exact mechanism of action of botulinum toxin type A (Botox) injections as a treatment for migraine are unknown, they are probably independent of the toxin's anti-cholinergic effects at the neuromuscular junction. It is no longer believed that the relaxation or induced weakness of muscles contributes to the therapeutic effect; instead inhibition of peripheral sensitization, leading to the inhibition of central sensitization through the blocking of glutamate, substance P and CGRP peripherally, is thought to lead to the therapeutic effect. There have been many positive open trials and a few double-blind, controlled studies with conflicting reports of efficacy.

In a chronic migraine trail, in which the primary endpoint was not reached, efficacy was shown only in a subgroup not taking preventive medication and having more than 15 headache days per month [11]. A recent study to compare the effectiveness of treatment of transformed migraine between botulinum toxin type A and topiramate demonstrated that both groups had significantly fewer headaches compared with baseline 6 months after the start of therapy. At 9 months, the two treatments were equivalent. More patients in the topiramate group dropped out of the study due to adverse events [12]. Another recent study compared botulinum toxin type A with divalproex in episodic and chronic migraine. The data demonstrate that both treatments showed a significant reduction in disability with fewer adverse events in the botulinum toxin type A treated group [13].

Two large, double-blind, placebo-controlled, randomized, phase III trials were performed in patients with transformed migraine, who were not on preventive medication. The results were released in Philadelphia at the International Headache Congress in September, 2009, a year after a press release was issued by Allergan [14] in September 2008 about the results. The primary endpoint for the first trial was the change from baseline in the 
number of headache episodes at the end of 3 months. In the second trial, the primary endpoint was the change in the number of headache days at the end of 3 months. At the request of Allergan, the FDA permitted a change in the primary endpoint in the second study prior to unlocking the data. The change was from the change from baseline in the number of headache episodes at 3 months to the change from baseline in the number of headache days (which is what the FDA had initially suggested for the primary endpoint).

The results showed that the first trial did not reach its primary endpoint and was negative. In the second trial, both the primary and secondary endpoints showed statistically significant benefit of botulinum toxin type A treatment over placebo injections. Patients treated with botulinum toxin type A demonstrated a greater decrease in both number of headache days $(p=0.001)$ (primary endpoint) and number of headache episodes $(p=0.003)$ (secondary endpoint). If the FDA suggested endpoint had been used (decrease in number of headache days), the first study would also have been positive. A meta-analysis of the two studies, one positive and one negative, was positive for all primary and secondary endpoints. Allergan has filed for an indication for the treatment of chronic migraine with botulinum toxin type $\mathrm{A}$ and expects a decision from the FDA by early 2011.

\section{Conclusion}

There are many new acute care and preventive therapies being investigated for the treatment of migraine. A few of them have been presented here. I am cautiously optimistic that some of the above treatments that are not yet available will make it to the clinic and will be effective additions to the headache specialist's armamentarium.

Conflict of interest statement A. Rapoport is on the Advisory Boards of NuPathe, MAP and Pfizer. He is a speaker for Endo, Forest, Merck, Pfizer and Zogenix and is an author of the Phase IIB study on telcagepant.

Open Access This article is distributed under the terms of the Creative Commons Attribution Noncommercial License which permits any noncommercial use, distribution, and reproduction in any medium, provided the original author(s) and source are credited.

\section{References}

1. Ramadan NM, Buchanan TM (2006) New and future migraine therapy. Pharmacol Ther 112:199-212

2. Goadsby PJ, Edvinsson L (1993) The trigeminovascular system and migraine: studies characterizing cerebrovascular and neuropeptide changes seen in humans and cats. Ann Neurol 33:48-56

3. Levy D, Burstein R, Strassman AM (2005) Calcitonin gene related peptide does not excite or sensitize meningeal nociceptors: implications for the pathophysiology of migraine. Ann Neurol 58:698-705

4. Olesen J, Diener HC, Husstedt IW et al (2004) Calcitonin gene related peptide receptor antagonist BIBN 4096 BS for the acute treatment of migraine. N Engl J Med 350:1104-1110

5. Ho TW, Mannix LK, Fan X et al (2008) Randomized controlled trial of an oral CGRP receptor antagonist, MK-0974, in acute treatment of migraine. Neurology 70:1304-1312

6. Ho TW, Ferrari MD, Dodick DW et al (2008) Efficacy and tolerability of MK-0974 (telcagepant), a new oral antagonist of calcitonin gene-related peptide receptor, compared with zolmitriptan for acute migraine: a randomised, placebo-controlled, parallel-treatment trial. Lancet 372:2089-2090

7. Rapoport AM (2009) Antimigraine drugs: new frontiers. Neurol Sci 30(Suppl 1):S49-S54 (some of the above text is borrowed in entirety from Neurol Sci)

8. Pierce M, Marbury T, O'Neill C et al (2008) A novel patch formulation of sumatriptan succinate utilizing SmartRelief ${ }^{\mathrm{TM}}$ transdermal technology. Data presented at the 50th annual scientific meeting of the American Headache Society 28 June 2008

9. Armer T, Shrewsbury S, Newman S et al (2007) Aerosol delivery of ergotamine tartrate via a breath-synchronized plume-control inhaler in humans. Curr Med Res Opin 23:3177-3187

10. Shrewsbury S, Cook R, Taylor G et al (2008) Safety and pharmacokinetics of dihydroergotamine mesylate administered via a novel (TEMPO_) Inhaler. Headache 48:355-367

11. Dodick DW, Mauskop A, Elkind AH et al (2005) Botulinum toxin type A for the prophylaxis of chronic daily headache: subgroup analysis of patients not receiving other prophylactic medications: a randomized double-blind, placebo-controlled study. Headache 45:315-324

12. Mathew NT (2008) A double-blind comparison of botulinum toxin type A (BoNTA) and topiramate for the prophylactic treatment of transformed migraine headaches: a pilot study. Presented at the 12th congress of the European Federation of the European Societies: 23-26 Aug 2008, Madrid, Spain

13. Blumenfeld AM, Schim JD, Chippendale TJ (2008) Botulinum toxin type A and divalproex sodium for prophylactic treatment of episodic or chronic migraine. Headache 48:210-220

14. Allergan announces positive top-line results from phase III BOTOX headache program-Released 11 Sept 20080900 a.m. Eastern Daylight Time 